\title{
Axion searches with Fermi and IACTs ${ }^{\text {ts }}$
}

\author{
Miguel A. Sánchez-Conde \\ Instituto de Astrofísica de Andalucía (CSIC), Camino Bajo de Huétor 50, E-18008, Granada, Spain
}

\begin{abstract}
Axion Like Particles (ALPs), postulated to solve the strong-CP problem, are predicted to couple with photons in the presence of magnetic fields, which may lead to a significant change in the observed spectra of gamma-ray sources such as AGNs. Here we 'simultaneously consider in the same framework both the photon/axion mixing that takes place in the gamma-ray source and that one 'expected to occur in the intergalactic magnetic fields. We show that photon/axion mixing could explain recent puzzles regarding the observed spectra of distant gamma-ray sources as well as the recently published lower limit to the EBL intensity. We finally summarize the different signatures expected and discuss the best strategy to search for ALPs with the Fermi satellite and current 'Cherenkov telescopes like CANGAROO, HESS, MAGIC and VERITAS.
\end{abstract}

Key words:

PACS: 95.35.+d, 95.55.Ka, 95.85.Pw, 98.70.Vc, 98.70.Rz, 14.80.Mz

\section{Photon/axion oscillations}

Axions were postulated to solve the CP problem in QCD in the 1970s [2]. Moreover, they are valid dark matter candidates to constitute a portion or the totality of the non-barionic cold dark matter content predicted to exist in the Universe. But probably the most interesting property of axions, or in a more generic way, Axion-Like Particles (ALPs), where the mass $m_{a}$ and the coupling constant are not related to each other, is that they are expected to oscillate into photons (and viceversa) in the presence of magnetic fields [3, 4]. This oscillation of photons to ALPs are the main vehicle used at present in axion searches, like those carried out by CAST [5] or ADMX [6], but they could also have important implications for astronomical observations. For example, they could distort the spectra of gamma'ray sources, such as Active Galactic Nuclei (AGNs) [7, 8, 9, 10] or galactic sources in the TeV range [11]. These distortions may be detected by current gamma-ray experiments, such as Imaging Atmospheric Cherenkov Telescopes (IACTs) like MAGIC [12], HESS [13], VERITAS [14] or CANGAROO-III [15], covering energies in the range $0.1-20 \mathrm{TeV}$, and by the Fermi satel-lite [16], which operates at energies in the range $0.02-300 \mathrm{GeV}$.

The probability of a photon of energy $E_{\gamma}$ to be converted into an ALP (or vice-versa) can be written as [7]:

$$
P_{0}=\frac{1}{1+\left(E_{c r i t} / E_{\gamma}\right)^{2}} \sin ^{2}\left[\frac{B s}{2 M} \sqrt{1+\left(\frac{E_{c r i t}}{E_{\gamma}}\right)^{2}}\right]
$$

where $s$ is the length of the domain where there is a roughly constant magnetic field, $\mathrm{B}$, and $M$ the inverse of the coupling

Talk given at RICAP 09, held in Villa Mondragone, Rome, Italy, in May 13-15, 2009, and based in the recent work by Sánchez-Conde et al. (Ref. [1]).

Email address: masc@iaa.es (Miguel A. Sánchez-Conde) constant. Here we also defined a characteristic energy, $E_{c r i t}$, which is equal to:

$$
E_{\text {crit }} \equiv \frac{m^{2} M}{2 B}
$$

or in more convenient units:

$$
E_{\text {crit }}(G e V) \equiv \frac{m_{\mu e V}^{2} M_{11}}{0.4 B_{G}}
$$

where the subindices refer to dimensionless quantities: $m_{\mu e V} \equiv$ $m / \mu e V, M_{11} \equiv M / 10^{11} \mathrm{GeV}$ and $B_{G} \equiv \mathrm{B} / \mathrm{Gauss} ; m$ is the effective ALP mass $m^{2} \equiv\left|m_{a}^{2}-\omega_{p l}^{2}\right|$, with $\omega_{p l}=\sqrt{4 \pi \alpha n_{e} / m_{e}}=$ $0.37 \times 10^{-4} \mu \mathrm{eV} \sqrt{n_{e} / \mathrm{cm}^{-3}}$ the plasma frequency, $m_{e}$ the electron mass and $n_{e}$ the electron density. Recent results from the CAST experiment [5] give a value of $M_{11} \geq 0.114$ for axion mass $m_{a} \leq 0.02 \mathrm{eV}$. Although there are other limits derived with other methods or experiments, the CAST bound is the most general and stringent limit in the range $10^{-11} \mathrm{eV} \ll m_{a} \ll 10^{-2} \mathrm{eV}$. In order to have an efficient conversion, we need:

$$
\frac{15 B_{G} s_{p c}}{M_{11}} \geq 1
$$

where $\mathrm{s}_{p c} \equiv \mathrm{s} /$ parsec. Some astrophysical environments fulfill the above mixing requirement and the $\mathrm{M}_{11}$ constraints imposed by CAST. Indeed, when using $M_{11} \geq 0.114$ in Eq. (4), we can deduce that astrophysical sources with $\mathrm{B}_{G} \cdot s_{p c} \geq 0.01$ will be valid. This product $\mathrm{B} \cdot s$ also determines the maximum energy $\mathrm{E}_{\text {max }}$ to which sources can accelerate cosmic rays, and is given by $\mathrm{E}_{\text {max }}=9.3 \times 10^{20} \mathrm{~B}_{G} \cdot s_{p c} \mathrm{eV}$ (Hillas criterium). Since we observe cosmic rays up to $3 \times 10^{20} \mathrm{eV}, \mathrm{B}_{G} \mathrm{~s}_{p c}$ can be as high as 0.3 , which also means that sources with $\mathrm{B}_{G} \cdot s_{p c}=0.01$ are completely plausible and should exist. Therefore, photon/axion mixing may have important implications for astronomical observations (AGNs, pulsars, GRBs...). Note, however, that an 
efficient mixing can be expected to occur not only in compact sources: the mixing will be also present in the Intergalactic Magnetic Fields (IGMFs), with typical values of $\sim 1 \mathrm{nG}$ for the B field, provided that the source is located at cosmological distances $\left(\mathrm{s}_{p c}=10^{8}\right)$ in order to ensure that $\mathrm{B} \cdot s$ is still larger than 0.01 .

Therefore, in order to correctly evaluate the photon/axion mixing effect for distant sources, it will be necessary to handle under the same consistent framework the mixing that takes place inside or near the gamma-ray sources together with that one expected to occur in the IGMF. In the literature, both effects have been considered separately. We neglect, however, the mixing that may happen inside the Milky Way due to galactic magnetic fields 1

\subsection{Mixing in the source}

To illustrate how the photon/axion mixing inside the source works, we show in Figure 1 an example for an AGN modeled by the parameters listed in Table 1. The only difference is the use of an ALP mass of $1 \mu \mathrm{eV}$ instead of the value that appear in that Table, so that we obtain a critical energy that lie in the $\mathrm{GeV}$ energy range; we get $E_{\text {crit }}=0.19 \mathrm{GeV}$ according to Eq. (3). Note that the main effect just above this critical energy is an attenuation in the total expected intensity of the source 2

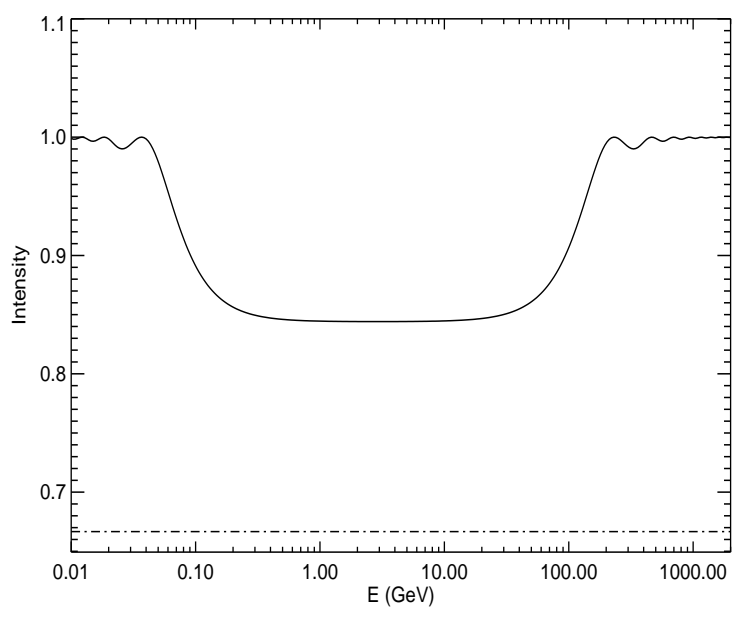

Figure 1: Example of photon/axion oscillations inside the source or vicinity, and its effect on the source intensity (solid line), which was normalized to 1 in the Figure. We used the parameters given in Table 1 to model the AGN source, but we adopted an ALP mass of $1 \mu \mathrm{eV}$. This gives $E_{\text {crit }}=0.19 \mathrm{GeV}$. The dot-dashed line represents the maximum (theoretical) attenuation, i.e. $1 / 3$.

\footnotetext{
${ }^{1}$ At present, a concise modeling of this effect is still very dependent on the largely unknown morphology of the magnetic field in the galaxy. Furthermore, in the most idealistic/optimistic case, it would produce a photon flux enhancement of $\sim 3 \%$ of the initial photon flux emitted by the source [10].

${ }^{2}$ Note also, however, that the attenuation starts to decrease at higher energies $(>10 \mathrm{GeV}$ ) gradually, the reason being the crucial role of the Cotton-Mouton term, which makes the efficiency of the source mixing to decrease as the energy increases. See Ref. [1] for details.
}

\subsection{Mixing in the IGMFs}

As already discussed, despite the low magnetic field $\mathbf{B}$, the photon/axion oscillation can take place in the IGMFs due to the large distances. However, the strength of the IGMFs is expected to be many orders of magnitude weaker $(\sim n G)$ than that of the source and its surroundings $(\sim \mathrm{G})$. Consequently, as described by Eq. (3), the energy at which photon/axion oscillation occurs in this case is many orders of magnitude larger than that at which oscillation can occur in the source and its vicinity. Assuming a mid-value of $\mathrm{B} \sim 0.1 \mathrm{nG}$, and $M_{11}=0.114$ (CAST upper limit), the effect could be observationally detectable by current IACTs $\left(E_{\text {crit }}<1 \mathrm{TeV}\right)$ only if the ALP mass is $\mathrm{m}_{a}<6 \times 10^{-10} \mathrm{eV}$, i.e. we need ultra-light ALPs. For example, we get $E_{\text {crit }}=28.5 \mathrm{GeV}$ when using $\mathrm{m}_{a}=10^{-10} \mathrm{eV}$, which is the value given in Table 1 as our reference one.

Table 1: Parameters used to calculate the total photon/axion conversion in both the source (for the two AGNs considered, 3c279 and PKS 2155-304) and in the IGM. The values related to 3C 279 were obtained from Ref. [17], while those ones for PKS 2155-304 were obtained from Ref. [18]. This Table represents our fiducial model.

\begin{tabular}{l|l|l|l} 
& Parameter & $3 \mathrm{C} 279$ & PKS 2155-304 \\
\hline \multirow{3}{*}{ Source } & $\mathrm{B}(\mathrm{G})$ & 1.5 & 0.1 \\
parameters & $\mathrm{e}_{d}\left(\mathrm{~cm}^{-3}\right)$ & 25 & 160 \\
& L domains $(\mathrm{pc})$ & 0.003 & $3 \times 10^{-4}$ \\
& B region $(\mathrm{pc})$ & 0.03 & 0.003 \\
\hline \multirow{3}{*}{$\begin{array}{l}\text { Intergalactic } \\
\text { parameters }\end{array}$} & $\mathrm{Z}$ & 0.536 & 0.117 \\
& $\mathrm{e}_{d, \text { int }}\left(\mathrm{cm}^{-3}\right)$ & $10^{-7}$ & $10^{-7}$ \\
& $\mathrm{~B}_{\text {int }}(\mathrm{nG})$ & 0.1 & 0.1 \\
\hline ALP & domains $(\mathrm{Mpc})$ & 1 & 1 \\
parameters & $\mathrm{M}(\mathrm{GeV})$ & $1.14 \times 10^{10}$ & $1.14 \times 10^{10}$ \\
& ALP mass $(\mathrm{eV})$ & $10^{-10}$ & $10^{-10}$
\end{tabular}

In our model, we assume that the photon beam propagates over $\mathrm{N}$ domains of a given length, the modulus of the magnetic field roughly constant in each of them. We take, however, randomly chosen orientations, which in practice is also equivalent to a variation in the strength of the component of the magnetic field involved in the photon/axion mixing 3 . Moreover, it will be necessary to include the effect of the Extragalactic Background Light (EBL) as well, its main effect being an additional attenuation of the photon flux, especially at energies above $100 \mathrm{GeV}$. Indeed, the EBL plays a crucial role to correctly evaluate and understand the importance of the intergalactic mixing. The induced effect can be an attenuation or an enhancement of the photon flux at Earth, depending on distance, magnetic field and the EBL model considered (see next Section).

In conclusion, AGNs located at cosmological distances will be affected by both mixing in the source and in the IGMF. Furthermore, and in order to observe both effects in the gammaray band, we need ultra-light ALPs. That is the reason why

\footnotetext{
${ }^{3}$ We refer to Ref. [1] for a detailed description of the model and the related equations.
} 


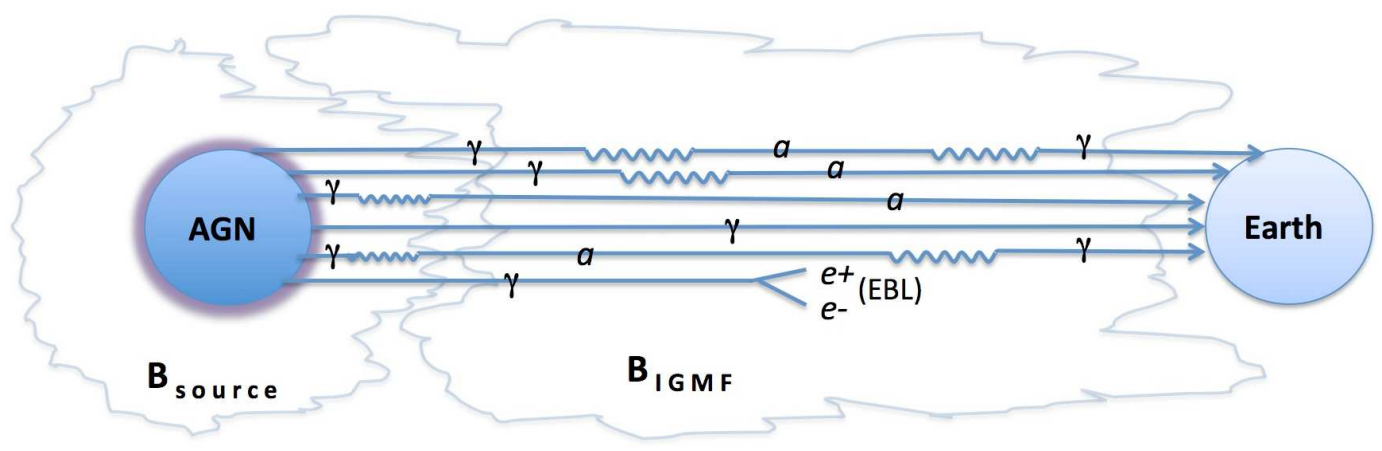

Figure 2: Sketch of the formalism here presented, where both mixing inside the source and mixing in the IGMF are considered under the same consistent framework. Photon to axion oscillations (or vice-versa) are represented by a crooked line, while the symbols $\gamma$ and $a$ mean gamma-ray photons and axions respectively. This diagram collects the main physical scenarios that we might identify inside our formalism. Each of them are squematically represented by a line that goes from the source to the Earth.

in our fiducial model, presented in Table 1 we use a value of $\mathrm{m}_{a}=10^{-10} \mathrm{eV}$, which implies $E_{\text {crit }} \sim 30 \mathrm{GeV}$ for the IGMF mixing (for $\mathrm{B} \sim 0.1 \mathrm{nG}$ ) and $E_{\text {crit }} \sim 1 \mathrm{eV}$ within the source and its vicinity $(\mathrm{B} \sim 1 \mathrm{G})$. Consequently, both effects need to be taken into account. We show in Fig. 2 a diagram that outlines our formalism. Very squematically, the diagram shows the travel of a photon from the source to the Earth in a scenario with ALPs. In the same Figure, we show the main physical cases that one could identify inside our formalism: mixing in both the source and the IGMF, mixing in only one of these environments, the effect of the EBL, axion to photon reconversions in the IGMF...

\section{Axion boosts}

Since we expect the intergalactic mixing to be more important for larger distances, due to the more prominent role of the EBL, we chose two distant astrophysical sources as our benchmark AGNs: the radio quasar 3C $279(\mathrm{z}=0.536)$ and the $\mathrm{BL}$ Lac PKS 2155-304 at $\mathrm{z}=0.117$. We summarize in Table 1 the parameters we have considered in order to calculate the total photon/axion conversion in both the source and in the intergalactic medium. As for the EBL model, we chose the Primack [19] and Kneiske best-fit [20] ones. They represent respectively one of the most transparent and one of the most opaque models for gamma-rays, but still within the limits imposed by the observations.

In order to quantitatively study the effect of ALPs on the total photon intensity, we plot in Figure 3 the difference between the predicted arriving photon intensity without including ALPs and that one obtained when including the photon/axion oscillations (called here the axion boost factor). This was done for our fiducial model (Table 1) and for the two EBL models considered. The plots show differences in the axion boost factors obtained for 3C 279 and PKS 2155-304 due mostly to the redshift difference. The inferred critical energies for the mixing in the source are $E_{\text {crit }}=4.6 \mathrm{eV}$ for $3 \mathrm{C} 279$ and $E_{\text {crit }}=69$ $\mathrm{eV}$ for PKS 2155-304, while for the mixing in the intergalactic medium we obtain $E_{\text {crit }}=28.5 \mathrm{GeV}$ (the same for both objects). For $\mathrm{B}=0.1 \mathrm{nG}$, and in the case of $3 \mathrm{C} 279$, the axion boost is an
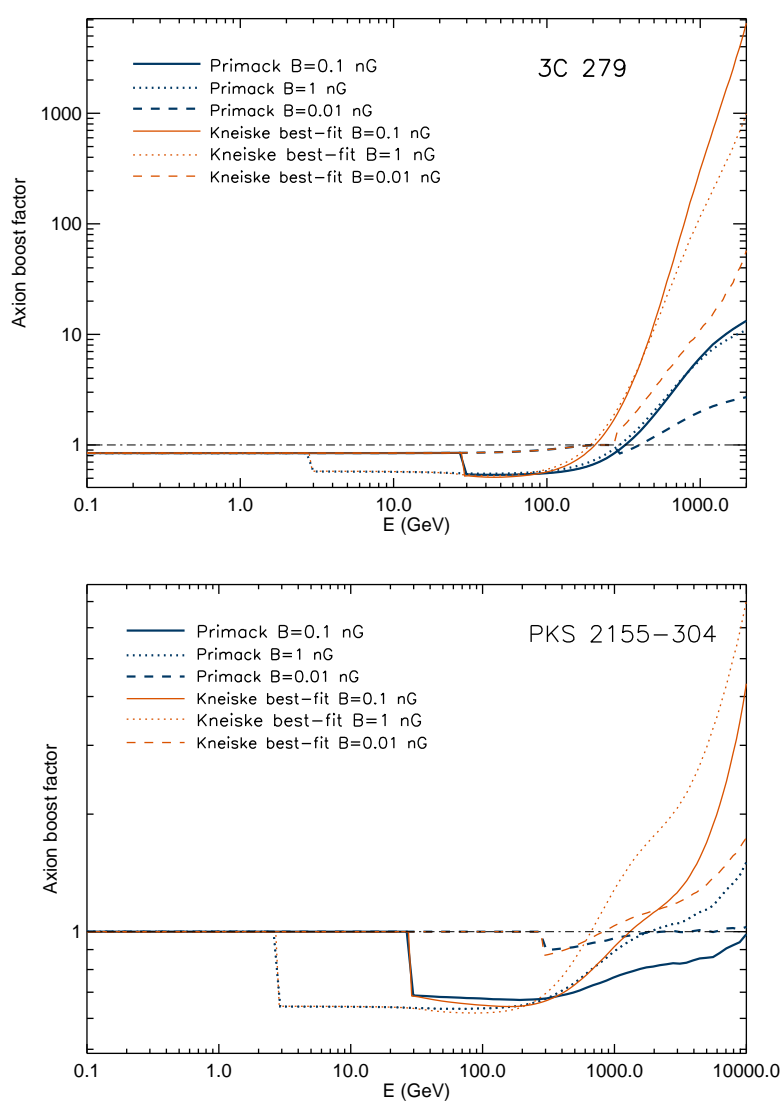

Figure 3: Boost in intensity due to ALPs for the Kneiske best-fit (red light lines) and Primack (blue dark lines) EBL models, and for different values of the magnetic field. We used those parameters presented in Table 1 for 3C $279(\mathrm{z}=0.536)$ and PKS 2155-304 ( $\mathrm{z}=0.117)$.

attenuation of about $16 \%$ below the critical energy (due to mixing inside the source). Above this critical energy and below 200-300 GeV, where the EBL attenuation is still small, there is an extra attenuation of about $30 \%$ (mixing in the IGMF). Above 200-300 GeV the axion boost reaches very high values: at 1 $\mathrm{TeV}$, a factor of $\sim 7$ for the Primack EBL model and $\sim 340$ for 


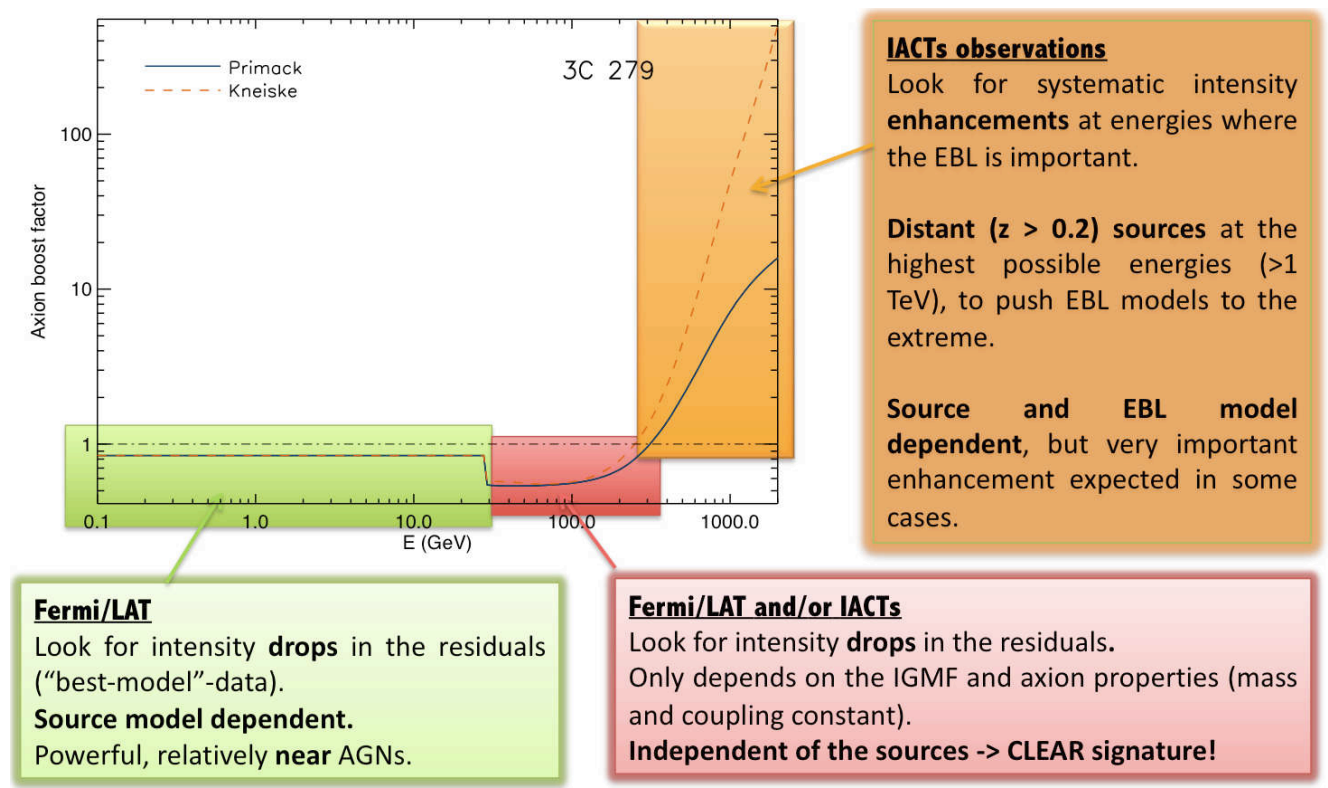

Figure 4: Summary of the best observational strategy to look for ALPs with Fermi and IACTs. See Ref. [1] for a deeper discussion.

the Kneiske best-fit model. We find that the more attenuating the EBL model considered, the more relevant the effect of photon/axion oscillations in the IGMF, since any ALP to photon reconversion might substantially enhance the intensity arriving at Earth. In the case of PKS 2155-304, the situation is different from that of $3 \mathrm{C} 279$ due to the very low photon-attenuation at the source and, mostly, due to the smaller source distance. Furthermore, a very interesting result is found when varying the modulus of the intergalactic magnetic field. Higher $\mathbf{B}$ values do not necessarily translate into higher photon flux enhancements. There is always a $\mathbf{B}$ value that maximizes the axion boost factors; this value is sensitive to the source distance, the considered energy and the EBL adopted model (see Ref. [1] for a detailed discussion on this issue).

\section{Detection prospects for Fermi and IACTs}

If we accurately knew the intrinsic spectrum of the sources and/or the density of the EBL, we should be able to observationally detect axion signatures or to exclude some portions of the parameter space. We lack this knowledge, so detection is challenging but we believe that still possible. The combination of the Fermi/LAT instrument and the IACTs, which cover 6 decades in energy (from $20 \mathrm{MeV}$ to $20 \mathrm{TeV}$ ) is very well suited to study the photon/axion mixing effect. Nevertheless, and before assuming an scenario with axions to interpret the observational data, we should try to describe the observational data (preferably several AGNs located at different redshifts, as well as the same AGN undergoing different flaring states, from radio to $\mathrm{TeV}$ ) with "conventional" theoretical models for the AGN emission and for the EBL. If these "conventional" models for the source emission and EBL fail (i.e. if we have important residuals for the best-fit model), then the axion scenario should be explored. Fig. 4 summarizes a good observational strategy to look for ALPs with Fermi and IACTs.
Recent gamma observations might already pose substantial challenges to the conventional models to explain the observed source spectra and/or EBL density [21, 22, 23, 24]. While it is still possible to explain these observations with conventional physics, the axion/photon oscillation would naturally explain these puzzles, since we get more high energy photons than expected as well as a harder observed spectrum in a scenario with ALPs.

\section{References}

[1] Sánchez-Conde M. A., Paneque D., Bloom E., Prada F. and Domínguez A., 2009, Phys. Rev. D, 79, 123511

[2] Peccei R. D. and Quinn H. R., 1977, Phys. Rev. Lett., 38, 1440

[3] Dicus D. A., Kolb E. W., Teplitz V. L. and Wagoner R. V., 1978, Phys. Rev. D, 18, 1829

[4] Sikivie P., 1983, Phys. Rev. Lett., 51, 1415 [Erratum ibid., 1984, Phys. Rev. Lett., 52, 695

[5] Andriamonje S. et al, 2007, JCAP, 0704, 010

[6] Duffy L. D. et al., 2006, Phys. Rev. D, 74, 012006

[7] Hooper D. and Serpico P., 2007, preprint, hep-ph/0706.3203

[8] De Angelis A., Roncadelli M. and Mansutti O., 2007, Phys. Rev. D, 76, 121301

[9] Hochmuth K. A., Sigl G., 2007, Phys. Rev. D, 76, 123011

[10] Simet M., Hooper D. and Serpico P., 2008, Phys. Rev. D, 77, 063001

[11] Mirizzi A., Raffelt G. G. and Serpico P., 2007, Phys. Rev. D, 76, 023001

[12] Lorentz E. et al., 2004, New Astron. Rev., 48, 339

[13] Hinton J. A., 2004, New Astron. Rev., 48, 331

[14] Weekes T. C. et al., 2002, Astropart. Phys., 17, 221

[15] Enomoto R. et al., 2002, Astropart. Phys., 16, 235

[16] Gehrels N. and Michelson P., 1999, Astropart. Phys., 11, 277

[17] Hartmann et al., 2001, ApJ, 553, 683

[18] Kusunose M. and Takahara F., 2008, ApJ, 682

[19] Primack J.R., 2005, Proceedings of the Gamma 2004 Symposium on High Energy Gamma Ray Astronomy, 26-30 July 2004, Heidelberg, Germany, astro-ph/0502177

[20] Kneiske T. M., Bretz T., Mannheim K. and Hartmann D. H., 2004, AE्FA, 413, 807

[21] Acciari V. A., 2009, ApJL, accepted, astro-ph/0901.4527

[22] Aliu et al., 2008, submitted to ApJL, astro-ph/0810.4712

[23] Levenson L. R. \& Wright E. L., 2008, ApJ, 683, 585

[24] Krennrich, Dwek and Imran, 2008, submitted to ApJ, astro-ph/0810.2522 